\title{
Accessible Multimodal Tool Support for Brainstorming Meetings
}

\author{
Reinhard Koutny ${ }^{1(\varpi)}\left(\mathbb{D}\right.$, Sebastian Günther ${ }^{2}\left(\mathbb{D}\right.$, Naina Dhingra ${ }^{3} \mathbb{D}$, \\ Andreas Kunz ${ }^{3}$, Klaus Miesenberger ${ }^{1}\left(\mathbb{D}\right.$, and Max Mühlhäuser ${ }^{2}(\mathbb{D}$ \\ 1 Institut Integriert Studieren, Johannes Kepler University, Linz, Austria \\ \{Reinhard.Koutny, Klaus.Miesenberger\}@jku.at \\ 2 Technische Universität Darmstadt, Darmstadt, Germany \\ \{guenther, max\}@tk.tu-darmstadt.de \\ 3 Innovation Center Virtual Reality, ETH Zurich, Zurich, Switzerland \\ \{ndhingra, kunz\}@iwf .mavt.ethz.ch \\ https://www.jku.at/institut-integriert-studieren/, \\ https://www.informatik.tu-darmstadt.de, \\ https://www.icvr.ethz.ch
}

\begin{abstract}
In recent years, assistive technology and digital accessibility for blind and visually impaired people (BVIP) has been significantly improved. Yet, group discussions, especially in a business context, are still challenging as non-verbal communication (NVC) is often depicted on digital whiteboards, including deictic gestures paired with visual artifacts. However, as NVC heavily relies on the visual perception, which represents a large amount of detail, an adaptive approach is required that identifies the most relevant information for BVIP. Additionally, visual artifacts usually rely on spatial properties such as position, orientation, and dimensions to convey essential information such as hierarchy, cohesion, and importance that is often not accessible to the BVIP. In this paper, we investigate the requirements of BVIP during brainstorming sessions and, based on our findings, provide an accessible multimodal tool that uses non-verbal and spatial cues as an additional layer of information. Further, we contribute by presenting a set of input and output modalities that encode and decode information with respect to the individual demands of BVIP and the requirements of different use cases.
\end{abstract}

Keywords: Blind and visually impaired people $\cdot$ Brainstorming · Business meeting $\cdot$ Non verbal communication $\cdot 2 \mathrm{D}$ haptic output device

\section{Introduction}

In recent years, the inclusion of persons with visual impairment or blindness has made significant strides towards an accessible society. Digital accessibility, in particular websites and applications found on stationary and mobile devices, has improved tremendously making the consumption of digital information, remote

(C) The Author(s) 2020

K. Miesenberger et al. (Eds.): ICCHP 2020, LNCS 12377, pp. 11-20, 2020.

https://doi.org/10.1007/978-3-030-58805-2_2 
communication and participation over the internet easier than ever before. In contrast, communication during in-situ meetings still heavily relies on non-verbal communication (NVC), which is hardly accessible to blind and visually impaired persons (BVIP) in most situations. In this context, sighted people do not only use their regular means of NVC, such as posture, gaze, facial expressions, or body language [22], but also make use of deictic gestures to refer to visual artifacts or other persons in the room during conversations [11]. While deictic gestures use arm and index finger for referencing points of interest [3,29] and are learned from childhood on $[4,6]$, this form of NVC is not self-contained and requires knowledge about where a speaker is pointing at as well as the information which person or artifact a person refers to $[1,7]$. Hence, spatial aspects, in particular the location of people and artifacts, play an important role. These spatial aspects are also often inaccessible to BVIP.

Thus, unrestricted participation in group conversations, which additionally make use of visual artifacts like cards or sketches on whiteboards or digital presentations, is still found to be a huge challenge for BVIP. Therefore, a good part of group conversations in a business context is inaccessible for BVIP. Also, tool support for brainstorming meetings has not adequately met the needs of BVIP. In particular, offering means to include a broad variety of user interaction approaches and devices to cater for a wide spectrum of users and use cases as well as considering accessibility has been a shortcoming in this area to date.

In order to address this shortcoming, we propose and implement dynamic tool support for BVIP during business meetings as part of the MAPVI project $[13]^{1}$. Thereby, we allow BVIP to fully participate by providing necessary non-verbal and spatial information as well as providing an accessible user interface to actively contribute on whiteboards. As proof-of-concept, we implement tool support in the form of a Metaplan application used during a brainstorming meeting with an ongoing group conversation.

In this paper, we investigate the requirements of BVIP during brainstorming meetings and present an accessible multimodal tool that utilizes non-verbal and spatial cues as an additional layer of information. We further contribute by presenting a set of in- and output modalities that encode and decode this information with regards to the individual demands of BVIP and requirements of the different use-cases.

The paper is structured as follows: Sect. 2 discusses related work. Section 3 describes the methodology. Section 4 explains the conceptual solution. Section 5 outlines the architecture. Section 6 gives an overview of user-interaction methods possible with this type of approach. Finally, Sect. 7 concludes the paper and gives an outlook on future work.

\footnotetext{
${ }^{1}$ The MAPVI project including this publication was founded by the Austrian Science Fund (FWF): I 3741-N31.
} 


\section{State of the Art}

Research investigated a large body of interactive systems which allow for bodybased [22] or gesture interaction $[8,35]$, as well as interfaces to allow intuitive world exploration techniques for BVIP $[5,10,15,34]$. However, during meeting situations, NVC has a tremendous amount of interaction possibilities that can enrich discussions for sighted persons during meeting situations, BVIP are often not able to fully access them and still rely on spoken information [13]. Further, business meetings nowadays usually make use of additional electronic tool support, such as digital presentations, and documents that can be collaboratively edited. Examples range from sticky notes [16] and mind map tools [21] to dedicated brainstorming and decision support software [12]. While all provide collaboration support, none of them provides specialized accessibility interfaces for BVIP.

To address these limitations, research has designed a variety of tools that allow for multimodal interfaces (e.g., $[2,17,24,31]$ ) and to support collaboration between BVIP and sighted people. For example, Thieme et al. [30] investigated how tools can assist during collaborative programming tasks. More recently, Shi et al. [28] presented a design tool for teachers to assist students with visual impairments to create interactive tactile maps. With regards to meetings, Regal et al. [27] presented an approach that uses NFC to map digital contents on tangible cards to support brainstorming meetings. Further, in a predecessor project [19], we investigated shortcomings of brainstorming meetings using mind maps and provided an accessible representation of them as an adaptive tree view for BVIP $[25,26]$. While this project already revealed great potential, it also exposed the need to support additional modalities to augment the perception of BVIP by providing further methods to handle NVC cues as well as spatial information within business meetings.

In summary, while there are a number of collaborative brainstorming tools that support BVIP through multimodal interfaces, there still remains a need in terms of business meetings, which helps BVIP to alleviate existing constraints that limit the access to non-verbal communication and spatial information, such as the location of participants, collaborative surfaces, or objects.

\section{Methodology}

User acceptance is one of the main success criteria of every project, tool or framework, therefore we involved users from the beginning of the project by interviews with ( 2 persons, both legally blind) and questionnaires (9 persons, 3 legally impaired and 6 visually impaired). According to these activities NVC is crucial in conversations, especially if more than two people are involved and that there might be disparity between NVC cues BVIP think are helpful during a first interview and after exposure to additional NVC cues. Observations show that the set of NVC cues perceived to be helpful is a very individual matter as well, pointing out the necessity of a highly customizable stream of information 


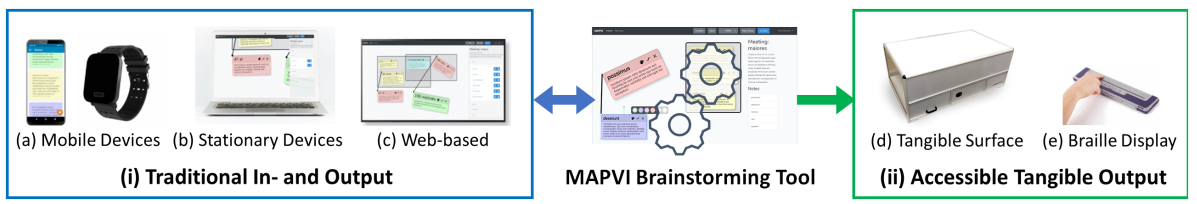

Fig. 1. Overview of the MAPVI brainstorming tool that supports (i) traditional inand output modalities, such as (a) mobile devices, (b) stationary devices and (c) webbased interfaces, as well as (ii) accessible tangible output modalities, such as (d) a magnetic surface with tangible representations and (e) modern braille displays.

conveying NVC cues. The outcomes of these efforts suggest that there is indeed a strong need for technologies, which can improve and support the perception of spatial aspects of group conversations and non-verbal communication for BVIP and a highly customizable solution would be greatly appreciated, since which parts of non-verbal communication are of most interest to a person and which level of detail is necessary and workable is a very individual matter depending on the person and the situation. According to these interviews and questionnaires, an ontology has been developed to describe the information space that is of relevance in this setting. Based on this foundation, the MAPVI brainstorming tool and its architecture were designed to allow for an accessible, extensible and customizable platform supporting a vast amount of devices and methods for user interaction to cater to an as broad as possible user group.

\section{Concept}

The MAPVI Brainstorming tool is developed to manage and support brainstorming meetings together with BVIP. The process and requirements of this application are derived from meeting sessions inspired by the Metaplan method. Therefore, it relies on different roles of participants, in particular a moderator and other participants, referred to as regular participants. These regular participants can be sighted people as well as BVIP. They can create notes, actively participate in the discussion and make decisions, finding consensus as a group. The moderator, on the other hand, asks questions, elicits and organizes ideas and encourages and directs discussions towards specific topics and subtopics arising during this creative process. However, the moderator does not create content or make decisions.

These major two roles are also reflected in the brainstorming tool (see Fig. 1). The moderator has his or her own user interface, which allow him or her to organize, place and group notes, created by regular participants. Moderators can also delete notes if requested by participants and agreed upon as a group. This is all done in a web-based application, which shows a virtual whiteboard consisting of notes, groups of notes and relations between notes. Since, whiteboards can 
contain numerous notes, which can quickly confuse users if presented in one view, multi-view functionality was implemented. This allows multiple views on the same whiteboard, which run simultaneously and show different parts of the whole whiteboard at different zoom levels. The role of regular participants is deliberately restricted to create notes and edit their own notes. This is done via a mobile application, which pushes new notes and changes of existing ones to a server, which is also accessed by the web-based whiteboard application.

\section{Architecture}

\subsection{Server-Backend}

The brainstorming tool is a client-server application, which consists of two data interfaces: a RESTful API to create, read, update, and delete data (CRUD) and a Web-Socket service for real-time notifications.

RESTful-API: Since access to data is not solely be done by one application or framework but multiple different ones, namely, at this stage from a web application and an Android application, a standardized approach is highly beneficial. Therefore, we decided to implement a RESTful-API, which allows for platformindependent, easy access to all necessary data. Via this API, applications of any kind can perform CRUD operations. In particular, data can be accessed concerning meetings in general, users, notes of meetings, groups of notes and relations between notes.

Authentication: Typically, this access needs to be restricted to people who are allowed to consume and modify data. To ensure this, we use a token-based authentication mechanism for the RESTful API to ensure the security and privacy of the data entered into the system.

Web-Sockets: Because multiple moderator views are possible as well as multiple Android clients can enter and modify data, data gets created, updated and deleted from multiple clients simultaneously. To keep the displayed information updated and consistent, we use Web-Sockets to inform all applications of changes in the data. Applications can register to specific channels, corresponding to data sets. If data gets changed on the server, the server broadcasts this information to appropriate channels and the clients will be informed about these changes.

\subsection{Web Interface}

The web interface is implemented with Laravel $^{2}$ using Bootstrap and the dynamic part is generated using Konva ${ }^{3}$ JavaScript Library. The web interface

\footnotetext{
${ }^{2}$ Laravel - PHP web framework: https://laravel.com.

${ }^{3}$ Konva - Javascript 2D Canvas Library: https://konvajs.org.
} 
displaying the whiteboard view accesses data via the RESTful API and listens to changes via Web-Sockets, for instance, when notes get created by participants using the Android app or get deleted in another instance of the whiteboard view.

\subsection{Android App}

The Android app accesses data using the RESTful API as well. Changes are communicated via Web-Sockets. Since the role of regular participants is deliberately restricted by the Metaplan technique, the purpose of the Android application, which is only used by regular participants, is very specific and the feature set is comparatively low. Essentially, the Android app allows adding new notes and modify notes that the user-created and it also gives an overview of all notes that were created by an individual user.

\section{Input and Output Modalities}

\subsection{General Accessibility of the User Interface}

The brainstorming tool supports a broad variety of user interaction methods. Again, the two different roles, moderator and regular participant, play an important role in this context. The moderator user interface, which is, as mentioned before, a web interface, can be operated using a mouse and keyboard, but it can also be operated using a touchscreen. Therefore, big touch screen devices acting as whiteboards are directly realizable. Also, multiple views on the same whiteboard are possible to show different parts of the whole composition of notes and other items. The selected architecture also allows for a keyboard-only operation, if moderators cannot use mice or touchscreens for whatever reason. Screenreader accessibility for the moderator user interface is possible too, because of the way that data is cached and updated in the browser using Web-Sockets.

\subsection{Recognition of NVC}

One part of the MAPVI project and this software suite is the inclusion of gesture recognition. So far pointing gestures of participants can be recognized [9]. The brainstorming tool allows these pointing gestures to be fed into the system and shared with BVIP. The architecture of the system allows because of the use of an easily extendable RESTful API the implementation of other NVC cues as well. Facial expressions, eye gestures and other hand gestures are possible in the future. This information can be later offered to BVIP.

\subsection{Gesture-Based User Interface for BVIP}

Additionally, gesture based user interfaces tailored for BVIP are supported, in particular, user interfaces considering spatial aspects of meetings. A mobile application using smartphones in combination with a smartwatch, which is currently 
under development, can be used to retrieve information about artifacts or participants by pointing in the general direction of information sources using the smartphone or smartwatch as virtual index finger, once this is finished. This will enable BVIP to easier select pieces of information, without cognitively overloading them and shows great potential to improve their spatial perception of the environment including the location of people and artifacts.

\subsection{Tangible Representation of the Information}

Tangibles provide a haptic experience and are able to map the content of individual items of the digital whiteboard to a physical surface and are distinguishable for BVIP by different shapes and tactile characteristics [14,18]. Further, they can maintain the position and clustering of the digital cards analogously. Hereby, it is important that the tangibles consistently adapt to the spatial information correctly, which requires an additional mechanism that can move them on the physical surface of the table, e.g., through embedded motors (e.g., [23]) or external mechanical support (e.g., [20]). As tangibles with embedded functionality may increase complexity and are expensive, external mechanisms that can move custom 3D-printed objects are reasonable. For example, electromagnetic arrays (e.g., $[32,33]$ or a magnetic gripper arm below the surface can precisely address tangibles with magnetic foundations individually and move each object according to the layout of the virtual whiteboard.

We implemented our prototype with a magnetic gripper arm to relocate tangibles to represent the digital content as cards. The spatial information of each card is maintained in real-time to make them physically accessible for BVIP and updates modifications of the spatial distribution of the cards dynamically. A top mounted camera detects the position of each tangible and recognizes changes by the user which triggers update commands to the tool. If a tangible is moved accidentally, the device can return it to its original location to keep the consistency. If a user touches a tangible with the index finger, the linked content of the virtual card of the brainstorming tool is readout.

\section{Conclusion and Further Work}

In this paper, we presented design considerations based on user involvement of members of the target group of BVIP and a proof-of-concept implementation of an accessible multimodal brainstorming tool for business meetings. A careful architectural design and selection of technology have ensured accessibility, extensibility, and customizability of this approach for brainstorming tool support. Multi-model user interface approaches including the tactile representation of information, gesture recognition or gesture-based user interfaces allow BVIP people to gain access to the vast information space of non-verbal communication and show great potential in helping them to participate in group conversations in a business context to the full extent. As the next step, existing user interface approaches will be refined and extended in close cooperation with people of the 
target group. In particular, gesture-based as well as tactile user interfaces will be investigated in more detail to explore how and to which degree they can help to deal with the immense amount of spatial and non-verbal information that gets generated during group conversations and prevent cognitive overloading blind and visually impaired people. Evaluations with the target group will be undertaken to study the benefits of this approach and reveal areas for improvements.

\section{References}

1. Alibali, M.W.: Gesture in spatial cognition: expressing, communicating, and thinking about spatial information. Spat. Cogn. Comput. 5(4), 307-331 (2005). https:// doi.org/10.1207/s15427633scc0504_2

2. Baldwin, M.S., Hayes, G.R., Haimson, O.L., Mankoff, J., Hudson, S.E.: The tangible desktop: a multimodal approach to nonvisual computing. ACM Trans. Access. Comput. 10(3) (2017). https://doi.org/10.1145/3075222

3. Bangerter, A., Oppenheimer, D.M.: Accuracy in detecting referents of pointing gestures unaccompanied by language. Gesture 6(1), 85-102 (2006). https://doi. org/10.1075/gest.6.1.05ban

4. Bates, E.: The Emergence of Symbols: Cognition and Communication in Infancy. Language, Thought, and Culture: Advances in the Study of Cognition. Academic Press, New York (1979). https://books.google.de/books?id=_45-AAAAMAAJ

5. Bolt, R.A.: Put-that-there: voice and gesture at the graphics interface. In: Proceedings of the 7th Annual Conference on Computer Graphics and Interactive Techniques, SIGGRAPH 1980, pp. 262-270. ACM, New York (1980). https://doi. org/10.1145/800250.807503

6. Brannigan, C.R., Humphries, D.A., Jones, B.: Ethological studies of child behaviour, pp. 37-64. Cambridge University Press, Cambridge (1972)

7. Butterworth, G., Jarrett, N.: What minds have in common is space: spatial mechanisms serving joint visual attention in infancy. Br. J. Dev. Psychol. 9(1), 55-72 (1991). https://doi.org/10.1111/j.2044-835X.1991.tb00862.x

8. Dhingra, N., Kunz, A.: Res3ATN-deep 3D residual attention network for hand gesture recognition in videos. In: 2019 International Conference on 3D Vision (3DV), pp. 491-501. IEEE (2019)

9. Dhingra, N., Valli, E., Kunz, A.: Recognition and localisation of pointing gestures using a RGB-D camera. arXiv preprint arXiv:2001.03687 (2020)

10. Geronazzo, M., Bedin, A., Brayda, L., Campus, C., Avanzini, F.: Interactive spatial sonification for non-visual exploration of virtual maps. Int. J. Hum.-Comput. Stud. 85, 4-15 (2016). https://doi.org/10.1016/j.ijhcs.2015.08.004. http://www. sciencedirect.com/science/article/pii/S1071581915001287. Data Sonification and Sound Design in Interactive Systems

11. Goffman, E.: Encounters: Two Studies in the Sociology of Interaction. Ravenio Books (1961)

12. Groupmap - collaborative brainstorming \& group decision-making (1442020). https://www.groupmap.com/

13. Günther, S., et al.: MAPVI: meeting accessibility for persons with visual impairments. In: Proceedings of the 12th PErvasive Technologies Related to Assistive Environments Conference, PETRA 2019, pp. 343-353. ACM, New York (2019). https://doi.org/10.1145/3316782.3322747 
14. Günther, S., Schmitz, M., Müller, F., Riemann, J., Mühlhäuser, M.: BYO*: utilizing 3D printed tangible tools for interaction on interactive surfaces. In: Proceedings of the 2017 ACM Workshop on Interacting with Smart Objects, SmartObject 2017, pp. 21-26. ACM, New York (2017). https://doi.org/10.1145/3038450.3038456

15. Guo, A., et al.: VizLens: a robust and interactive screen reader for interfaces in the real world. In: Proceedings of the 29th Annual Symposium on User Interface Software and Technology, UIST 2016, pp. 651-664. ACM, New York (2016). https:// doi.org/10.1145/2984511.2984518

16. Ideaflip - realtime brainstorming and collaboration (1442020). https://ideaflip. $\mathrm{com} /$

17. Iranzo Bartolome, J., Cavazos Quero, L., Kim, S., Um, M.Y., Cho, J.: Exploring art with a voice controlled multimodal guide for blind people. In: Proceedings of the Thirteenth International Conference on Tangible, Embedded, and Embodied Interaction, TEI 2019, pp. 383-390. Association for Computing Machinery, New York (2019). https://doi.org/10.1145/3294109.3300994

18. Ishii, H.: Tangible bits: beyond pixels. In: Proceedings of the 2nd International Conference on Tangible and Embedded Interaction, TEI 2008, pp. xv-xxv. Association for Computing Machinery, New York (2008). https://doi.org/10.1145/1347390. 1347392

19. Kunz, A., et al.: Accessibility of brainstorming sessions for blind people. In: Miesenberger, K., Fels, D., Archambault, D., Peňáz, P., Zagler, W. (eds.) ICCHP 2014. LNCS, vol. 8547, pp. 237-244. Springer, Cham (2014). https://doi.org/10.1007/ 978-3-319-08596-8_38

20. Lee, J., Post, R., Ishii, H.: ZeroN: mid-air tangible interaction enabled by computer controlled magnetic levitation. In: Proceedings of the 24th Annual ACM Symposium on User Interface Software and Technology, UIST 2011, pp. 327-336. Association for Computing Machinery, New York (2011). https://doi.org/10.1145/ 2047196.2047239

21. Miro - mind map software built with teams in mind (1442020). https://miro.com/

22. Müller, C., Cienki, A., Fricke, E., Ladewig, S., Mcneill, D., Tessendorf, S.: Body - Language - Communication: An International Handbook on Multimodality in Human Interaction, vol. Bd. 38.1. De Gruyter Inc., Berlin/Boston (2013)

23. Pedersen, E.W., Hornbæk, K.: Tangible bots: interaction with active tangibles in tabletop interfaces. In: Proceedings of the SIGCHI Conference on Human Factors in Computing Systems, CHI 2011, pp. 2975-2984. Association for Computing Machinery, New York (2011). https://doi.org/10.1145/1978942.1979384

24. Pires, A.C., et al.: A tangible math game for visually impaired children. In: The 21st International ACM SIGACCESS Conference on Computers and Accessibility, ASSETS 2019, pp. 670-672. Association for Computing Machinery, New York (2019). https://doi.org/10.1145/3308561.3354596

25. Pölzer, S., Miesenberger, K.: Presenting non-verbal communication to blind users in brainstorming sessions. In: Miesenberger, K., Fels, D., Archambault, D., Peňáz, P., Zagler, W. (eds.) ICCHP 2014. LNCS, vol. 8547, pp. 220-225. Springer, Cham (2014). https://doi.org/10.1007/978-3-319-08596-8_35

26. Pölzer, S., Miesenberger, K.: A tactile presentation method of mind maps in colocated meetings. In: Proceedings of the International Workshop on Tactile/Haptic User Interfaces for Tabletops and Tablets, Held in Conjunction with ACM ITS 2014. ACM, New York (2014)

27. Regal, G., Mattheiss, E., Sellitsch, D., Tscheligi, M.: TalkingCards: using tactile NFC cards for accessible brainstorming. In: Proceedings of the 7th Aug- 
mented Human International Conference 2016, AH 2016. Association for Computing Machinery, New York (2016). https://doi.org/10.1145/2875194.2875240

28. Shi, L., Zhao, Y., Gonzalez Penuela, R., Kupferstein, E., Azenkot, S.: Molder: an accessible design tool for tactile maps. In: Proceedings of the 2020 CHI Conference on Human Factors in Computing Systems, CHI 2020, pp. 1-14. Association for Computing Machinery, New York (2020). https://doi.org/10.1145/3313831. 3376431

29. Taylor, J.L., McCloskey, D.I.: Pointing. Behav. Brain Res. 29(1), 1-5 (1988). https://doi.org/10.1016/0166-4328(88)90046-0

30. Thieme, A., Morrison, C., Villar, N., Grayson, M., Lindley, S.: Enabling collaboration in learning computer programing inclusive of children with vision impairments. In: Proceedings of the 2017 Conference on Designing Interactive Systems, DIS 2017, pp. 739-752. Association for Computing Machinery, New York (2017). https://doi.org/10.1145/3064663.3064689

31. Yu, W., Kangas, K., Brewster, S.: Web-based haptic applications for blind people to create virtual graphs. In: 2003 Proceedings of the 11th Symposium on Haptic Interfaces for Virtual Environment and Teleoperator Systems, HAPTICS 2003, pp. 318-325 (2003)

32. Weiss, M., Remy, C., Borchers, J.: Rendering physical effects in tabletop controls. In: Proceedings of the SIGCHI Conference on Human Factors in Computing Systems, CHI 2011, pp. 3009-3012. Association for Computing Machinery, New York (2011). https://doi.org/10.1145/1978942.1979388

33. Weiss, M., Schwarz, F., Jakubowski, S., Borchers, J.: Madgets: actuating widgets on interactive tabletops. In: Proceedings of the 23nd Annual ACM Symposium on User Interface Software and Technology, UIST 2010, pp. 293-302. Association for Computing Machinery, New York (2010). https://doi.org/10.1145/1866029.1866075

34. Willis, S., Helal, S.: RFID information grid for blind navigation and wayfinding. In: Ninth IEEE International Symposium on Wearable Computers (ISWC 2005), pp. 34-37. IEEE, New York (2005). https://doi.org/10.1109/ISWC.2005.46

35. Zhai, S., Morimoto, C., Ihde, S.: Manual and gaze input cascaded (magic) pointing. In: Proceedings of the SIGCHI Conference on Human Factors in Computing Systems, CHI 1999, pp. 246-253. ACM, New York (1999). https://doi.org/10.1145/ 302979.303053

Open Access This chapter is licensed under the terms of the Creative Commons Attribution 4.0 International License (http://creativecommons.org/licenses/by/4.0/), which permits use, sharing, adaptation, distribution and reproduction in any medium or format, as long as you give appropriate credit to the original author(s) and the source, provide a link to the Creative Commons license and indicate if changes were made.

The images or other third party material in this chapter are included in the chapter's Creative Commons license, unless indicated otherwise in a credit line to the material. If material is not included in the chapter's Creative Commons license and your intended use is not permitted by statutory regulation or exceeds the permitted use, you will need to obtain permission directly from the copyright holder.



